\title{
Efeito de fontes de óleo e níveis de suplementação de vitamina E na ração sobre as características físicas e morfológicas do sêmen in natura de suínos
}

\author{
[Effect of oil sources and vitamin E supplementation in the diet on physical and morphological characteristics of \\ fresh boar semen] \\ E.A. Moraes $^{1}$, C.A.A. Torres ${ }^{2}$, J.D. Guimarães ${ }^{3}$, L.D.S. Murgas ${ }^{4}$ \\ ${ }^{1}$ Colegiado de Zootecnia - UNIVASF \\ Av. José de Sá Maniçoba, s/n \\ 56304-205 - Petrolina, PE \\ ${ }^{2}$ Departamento de Zootecnia - UFV - Viçosa, MG \\ ${ }^{3}$ Departamento de Veterinária - UFV - Viçosa, MG \\ ${ }^{4}$ Departamento de Veterinária - UFLA - Lavras, MG
}

\begin{abstract}
RESUMO
Avaliou-se o efeito da adição de diferentes fontes de óleo e níveis de suplementação de vitamina E na ração sobre as características do sêmen in natura de suínos reprodutores. Foram utilizados 24 reprodutores Dalboar 85, com idades entre 12 e 18 meses, distribuídos em delineamento inteiramente ao acaso, em arranjo fatorial 2 x 3, com duas fontes de óleo, soja e salmão, e três níveis de antioxidantes, 150, 300 e 450mg de vitamina E/kg. Volume, motilidade espermática total, teste hiposmótico, porcentagem de espermatozoides vivos e morfologia espermática não diferiram $(\mathrm{P}>0,05)$ entre os tratamentos. Óleo de salmão $(\mathrm{P}<0,05)$ melhorou o vigor espermático. A inclusão de vitamina $E$ na ração melhorou $(\mathrm{P}<0,05)$ a concentração espermática, e não foi observada diferença entre as fontes de óleo $(\mathrm{P}>0,05)$. Os animais tratados com óleo de salmão apresentaram menor $(\mathrm{P}<0,05)$ concentração de antioxidantes totais no sêmen do que os tratados com óleo de soja. Observouse efeito linear da vitamina $E$ sobre a concentração de antioxidantes totais $(\mathrm{P}<0,05)$. A fonte de óleo de salmão da ração melhora o vigor espermático e a concentração de antioxidantes totais no sêmen.
\end{abstract}

Palavras-chave: varrão, ácidos graxos, antioxidantes, características seminais

\begin{abstract}
The addition of oil sources and dietary vitamin E supplementation was evaluated on the characteristics of fresh boar sperm. Twenty-four mature Dalboar 85 boars of proven fertility and in routine semen production for artificial insemination were randomly distributed in a factorial arrangement $2 X 3$, with two oil sources, soy and salmon, and three antioxidant levels: 150,300 , and $450 \mathrm{mg} / \mathrm{kg}$ of vitamin E. Volume, total motile sperm, hyposmotic swelling test, percentage of live cell, and morphology did not differ $(P<0.05)$ between the animals from the treatments. Salmon oil improved $(P<0.05)$ sperm vigor. The vitamin $E$ added to the diet improved $(P<0.05)$ the sperm concentration, and there was no difference between the oil sources $(P>0.05)$. Salmon oil treatment showed the lowest $(P<0.05)$ concentration of total antioxidants in the semen. Vitamin $E$ and concentration of total antioxidant showed a linear effect $(P<0.05)$. The semen vigor and the concentration of total antioxidants were improved by oil sources, but the sperm concentration was dependent on the levels of vitamin E. Therefore, salmon oil can be used in the diet of male pigs.
\end{abstract}

Keywords: boar, fatty acid, total antioxidant, seminal characteristics

\section{INTRODUÇÃO}

Na membrana das células, os ácidos graxos são elementos estruturais e compostos bioativos. Em muitas espécies, os fosfolipídios são os principais componentes lipídicos dos espermatozoides, caracterizados por terem grande quantidade de ácidos graxos poliinsaturados. Em alguns mamíferos, o ácido docosa-hexanoico é o ácido graxo poliinsaturado dominante, embora em outras espécies o ácido docosapentanoico seja o

Recebido em 8 de julho de 2009

Aceito em 3 de maio de 2010

E-mail: eamufv@yahoo.com.br 
principal componente da membrana espermática. O sêmen suíno apresenta ambos os ácidos graxos em grande quantidade (Penny et al., 2000). Com relação à constituição da membrana celular, o aumento na proporção de poli-insaturados nos espermatozoides, durante sua fase de maturação no epidídimo, pode ter efeito pronunciado nas propriedades físicas e químicas da membrana. Desse modo, a inclusão de óleos na ração pode modificar o perfil de ácidos graxos dos espermatozoides e, consequentemente, melhorar a qualidade espermática (Scott, 1973). Os ácidos graxos poli-insaturados da série ômega $3(\omega 3)$ predominam nos fosfolipídios das membranas celulares de cuja permeabilidade e flexibilidade dependem (Sirri et al. 1995).

Mesmo apresentando efeitos benéficos nos ácidos graxos $\omega 3$, a presença de alta concentração de ácidos graxos poli-insaturados dentro das frações lipídicas dos espermatozoides torna-os altamente susceptíveis à peroxidação, com consequente risco de danos à estrutura celular (Niki et al., 1993). Portanto, há necessidade de um sistema antioxidante para protegê-los contra danos peroxidativos bem como contra uma possível disfunção dessas células (Cecil e Bakst, 1993; Aitken, 1994). Acredita-se que os danos causados pela peroxidação dos lipídios dos espermatozoides sejam a principal causa de subfertilidade dos machos (Hammerstedt, 1993). Assim, este trabalho teve o objetivo de avaliar o efeito de diferentes fontes de óleo e de níveis de suplementação de vitamina $\mathrm{E}$ sobre as características do sêmen in natura de suínos.

\section{MATERIAL E MÉTODOS}

O experimento foi realizado de setembro de 2005 a janeiro de 2006. Foram utilizados 24 suínos machos reprodutores Dalboar 85, com idades entre 12 e 18 meses, alojados em baias individuais com água ad libitum e suplementação concentrada diária, segundo as exigências estabelecidas pelo NRC (Nutrient..., 1998). Os animais foram distribuídos em um delineamento inteiramente ao acaso, em arranjo fatorial $2 \times 3$, com duas fontes de óleo, soja e salmão, e três níveis de antioxidantes, 150, 300 e 450mg de vitamina $\mathrm{E} / \mathrm{kg}$ de ração, durante 10 semanas. Foram adicionados $3,5 \%$ de óleo, independentemente da fonte utilizada. A composição centesimal da ração, baseada nas exigências nutricionais do NRC (Nutrient..., 1998), encontra-se na Tab. 1.

As amostras de sêmen foram coletadas, semanalmente, uma coleta/macho/semana, pelo método da mão enluvada (King e MacPherson, 1973), em sala apropriada com um manequim. O sêmen foi coletado em copo térmico, com água a $37^{\circ} \mathrm{C}$. Imediatamente após a coleta do sêmen, o ejaculado foi analisado quanto ao volume $(\mathrm{mL})$, à concentração espermática $\left(\times 10^{6} / \mathrm{mL}\right)$, à motilidade espermática total, ao vigor espermático, à porcentagem de espermatozoides vivos e mortos, à morfologia espermática e ao teste hiposmótico, conforme normas do CBRA (Manual..., 1998).

O volume espermático foi determinado por meio da pesagem do ejaculado sem a fração gelatinizada, em que $1 \mathrm{~mL}$ do ejaculado pesa, aproximadamente, $1 \mathrm{~g}$.

Tabela 1. Composição centesimal da ração experimental disponibilizada para reprodutores suínos

\begin{tabular}{lc}
\hline Ingrediente & Quantidade (kg) \\
\hline Milho & 66,05 \\
Farelo de soja & 19,40 \\
Casca de soja & 7,38 \\
Óleo $^{1}$ & 3,50 \\
Foscálcio $_{\text {Calcário }}$ & 1,63 \\
Sal & 0,87 \\
Premix vitamina e mineral & 2,45 \\
Adsorvente Mycofix & 0,40 \\
Lisina & 0,20 \\
Total & 0,13 \\
\hline Composição calculada & 100,00 \\
\hline Matéria seca (\%) & \\
Energia metabolizável (kcal/kg) & 87,53 \\
Proteína bruta (\%) & 3.555 \\
Fibra (\%) & 17,3 \\
Cálcio (\%) & 4,60 \\
Fósforo disponível (\%) & 0,79 \\
Gordura (\%) & 0,45 \\
Metionina (\%) & 6,59 \\
Metionina + cistina (\%) & 0,237 \\
Lisina (\%) & 0,503 \\
\hline
\end{tabular}

${ }^{\mathrm{T}}$ Óleos de soja e de salmão foram adicionados na mesma proporção.

${ }^{2}$ Premix vitamínico e mineral suíno reprodução $0,4 \%$ (Perdigão S/A - Cascavel, Brasil).

A concentração espermática foi determinada pelo aparelho espectrofotômetro SpermaCue (Minitub do Brasil Ltda.), que utiliza um filtro de 570nm para medir o grau de opacidade da amostra. Para 
a análise, $20 \mu \mathrm{L}$ da amostra do sêmen foram retirados e colocados na microcuveta, que apresenta a superfície interna revestida com um reagente seco, e a contagem foi realizada pelo número de células por unidade de volume estimado pela opacidade do ejaculado, a qual foi medida pela porcentagem de transmissão ou absorbância da amostra.

A avaliação da motilidade total (\%) e do vigor espermático foi examinada em microscopia de contraste de fase em aumento de 200x, utilizando-se uma alíquota de $10 \mu \mathrm{L}$ de sêmen, depositada entre lâmina e lamínula, ambas mantidas a $37^{\circ} \mathrm{C}$. O vigor foi classificado de acordo com Ferreira Neto et al. (1977), variando de zero (0) a cinco (5), sendo o escore 0 equivalente à total imobilidade espermática, e o escore 5 à movimentação intensa, vigorosa, progressiva e com formação de ondas.

A coloração foi realizada imediatamente após a coleta. Foram misturadas sete gotas de corante eosina-nigrosina com três gotas de sêmen in natura em um tubo pequeno (Murgas et al., 2001). O esfregaço foi feito em lâmina aquecida e examinado em microscópio com aumento de 400x para identificar os espermatozoides vivos (aparecendo sem coloração) e os mortos que absorveram o corante e se tornaram vermelhos, segundo metodologia de Barth e Oko (1989). Foram contadas 100 células espermáticas de cada amostra.

Para a avaliação da morfologia espermática, utilizou-se a técnica de preparação úmida. Alíquotas de $10 \mu \mathrm{L}$ de sêmen foram adicionadas em $1 \mathrm{~mL}$ de formol-salino tamponado e estocado à temperatura ambiente até análises. Foram contadas 100 células no microscópio óptico de contraste de fase no aumento de 1000x para a determinação do percentual de anormalidades espermáticas, classificadas segundo CBRA (Manual..., 1998), e dividiram-se as anormalidades em defeitos maiores e menores.

O teste hiposmótico (HOST), que avalia a integridade da membrana plasmática, foi usado no sêmen in natura, conforme a técnica da preparação úmida. Na solução à base de citrato de sódio e frutose $(0,9 \mathrm{~mL})$, com osmolaridade de 150mOsmol/L (Vazquez et al., 1997), foram adicionados $10 \mu \mathrm{L}$ de sêmen diluído, e a mistura foi incubada por 30 minutos, em banho-maria a $37^{\circ} \mathrm{C}$. Após esse período, alíquotas de $15 \mu \mathrm{L}$ da mistura foram colocadas entre lâmina e lamínula, para a contagem de 100 células de cada solução em microscopia de contraste de fase com aumento de 400x. As células foram classificadas quanto à presença ou não da cauda enrolada, segundo Kumi-Diaka (1993).

A avaliação in vitro da concentração de antioxidantes totais (AOT) no sêmen foi feita em ejaculados de suínos na primeira, quarta, sétima e décima semanas, utilizando-se kits (Randox Limited, Crumlin) apropriados. Foram avaliadas 100 amostras, distribuídas entre os tratamentos.

As análises foram realizadas com a utilização do programa SAEG (Sistema..., 1997). As variáveis foram submetidas aos testes de normalidade e homocedacidade e, posteriormente, à análise de variância e ao teste de SNK para comparação de médias entre as fontes de óleo, com nível de significância de 5\%, e à análise de regressão para os níveis de antioxidantes usados. Quando houve interação, foi determinado o efeito do nível de antioxidante dentro de cada fonte. As variáveis que não atenderam às premissas para a análise de variância, mesmo após as transformações, foram submetidas ao teste não paramétrico de Wilcoxon $(\mathrm{P}<0,05)$.

\section{RESULTADOS E DISCUSSÃO}

Não foram observados efeitos da fonte de óleo e do nível de vitamina $\mathrm{E}$ nem de sua interação sobre o volume do sêmen, a motilidade espermática total e a porcentagem de espermatozoides vivos (Tab. 2), sobre a porcentagem de espermatozoides reativos ao HOST (Tab. 3) e sobre a morfologia espermática (Tab. 4) (P>0,05). Os valores médios das características seminais observadas aproximam-se dos padrões de normalidade considerados para a espécie estudada (Henry e Neves, 1998). Zanini et al. (2003a) testaram diferentes fontes de óleo na dieta de galos, cuja membrana espermática é rica em ômega 6 , e observaram aumento no volume do sêmen dos galos suplementados com óleo de soja, não diferindo dos tratados com óleo de peixe.

Os resultados de motilidade espermática total, porcentagem de espermatozoides vivos e morfologia anormal diferem dos observados por Maldjian et al. (2005), que verificaram o efeito 
da suplementação na ração de suínos com ácido docosa-hexanoico (DHA) versus outra sem DHA. Observou-se que a correlação entre a motilidade espermática e o teor de DHA nos espermatozoides não foi alta nos tratamentos com óleo de soja $(\mathrm{r}=0,61)$ e óleo de salmão $(\mathrm{r}=0,53)$. Nissen e Kreysel (1983), Gulaya et al. (1993) e Conquer et al. (2000) relataram que o teor de DHA nos espermatozoides humanos está correlacionado com a motilidade espermática. Não se sabe como o DHA pode estar envolvido na regulação da motilidade espermática em humanos, talvez por estar envolvido na regulação de ácidos graxos livres utilizados pelo espermatozoide (Jones e Plymate, 1988; 1993) ou na metabolização de outros compostos lipídicos (Oliw e Sprecher, 1991). As propriedades biofísicas do DHA contribuem com a fluidez e a flexibilidade da membrana necessárias para a motilidade da cauda (Connor et al., 1998).

Tabela 2. Volume do sêmen, motilidade e porcentagem de espermatozoides vivos no sêmen in natura de suínos, de acordo com a fonte de óleo e o nível de suplementação de vitamina $\mathrm{E}$

\begin{tabular}{|c|c|c|c|c|c|}
\hline \multirow[t]{2}{*}{ Fonte de óleo } & \multicolumn{4}{|c|}{ Nível de vitamina $\mathrm{E}(\mathrm{mg} / \mathrm{kg})$} & \multirow[b]{2}{*}{$\mathrm{CV}(\%)$} \\
\hline & 150 & 300 & 450 & Média & \\
\hline \multicolumn{6}{|c|}{ Volume de sêmen } \\
\hline Soja & 200,6 & 249,7 & 205,9 & 218,7 & \\
\hline Salmão & 223,9 & 227,5 & 219,4 & 223,6 & \\
\hline Média & 212,3 & 238,6 & 212,7 & & 38,3 \\
\hline \multicolumn{6}{|c|}{ Motilidade espermática } \\
\hline Soja & 83,7 & 85,0 & 85,1 & 84,6 & \\
\hline Salmão & 85,0 & 84,4 & 85,2 & 84,9 & \\
\hline Média & 84,4 & 84,7 & 85,2 & & 3,63 \\
\hline \multicolumn{6}{|c|}{ Espermatozoides vivos } \\
\hline Soja & 85,3 & 86,1 & 85,7 & 85,7 & \\
\hline Salmão & 85,8 & 85,9 & 85,7 & 85,8 & \\
\hline Média & 85,5 & 86,0 & 85,7 & & 2,26 \\
\hline
\end{tabular}

Não houve diferença entre as médias pelo teste SNK (P>0,05).

O HOST avalia a integridade funcional da membrana plasmática (baseado nas propriedades osmóticas da membrana plasmática), enquanto o teste de coloração mostra a integridade estrutural da membrana espermática (baseado na impermeabilidade de grandes moléculas de corantes). Espermatozoides vivos podem ter a membrana não funcional, incapaz de reagir (dobramento da cauda), ainda que suas estruturas estejam intactas. Por isso, o HOST pode ser um teste mais sensível do que o teste de coloração. Em ambas as fontes de óleo adicionadas às rações, os espermatozoides foram altamente reativos ao teste hiposmótico, mostrando que não há perda da funcionalidade na membrana plasmática com o uso desses óleos.

Os valores registrados para o HOST (Tab. 3) corroboram com os de Vazquez et al. (1997), que adaptaram o HOST para espermatozoides de suíno, e reportaram ser este um teste de alta sensibilidade e repetibilidade para avaliar a integridade funcional da membrana plasmática de suínos.

Tabela 3. Valores do teste hiposmótico no sêmen in natura de suínos, de acordo com a fonte de óleo e o nível de suplementação de vitamina $\mathrm{E}$

\begin{tabular}{|c|c|c|c|c|c|}
\hline \multirow{2}{*}{ Fonte de óleo } & \multicolumn{4}{|c|}{ Nível de vitamina $\mathrm{E}(\mathrm{mg} / \mathrm{kg})$} & \multirow[b]{2}{*}{$\mathrm{CV}(\%)$} \\
\hline & 150 & 300 & 450 & Média & \\
\hline Soja & 82,8 & 86,0 & 83,9 & 84,2 & \\
\hline Salmão & 83,5 & 86,2 & 88,8 & 86,2 & \\
\hline Média & 83,2 & 86,1 & 86,4 & & 14,4 \\
\hline
\end{tabular}

Não houve diferença entre as médias pelo teste SNK $(\mathrm{P}>0,05)$. 
Para vitaminas lipossolúveis, a vitamina E pode estar envolvida na proteção direta das células espermáticas de danos morfológicos (Brzezinska-Slebodzinska et al., 1995). De fato, a morfologia e a motilidade espermática total dos espermatozoides podem ser preservadas por meio da ligação dessas vitaminas a endoperóxidos (Marin-Guzman et al., 1997;
2000). A porcentagem máxima de anomalias espermáticas em espermatozoides de reprodutores suínos é de 20\% (Manual..., 1998), portanto as adições de óleo de soja ou de salmão e a de vitamina $E$ à ração não afetaram a qualidade do sêmen, ou seja, a morfologia espermática (Tab. 4).

Tabela 4. Defeitos maiores e menores observados nos espermatozoides no sêmen in natura de suínos, de acordo com a fonte de óleo e o nível de suplementação de vitamina $\mathrm{E}$

\begin{tabular}{|c|c|c|c|c|c|}
\hline \multirow{2}{*}{ Fonte de óleo } & \multicolumn{4}{|c|}{ Nível de vitamina $\mathrm{E}(\mathrm{mg} / \mathrm{kg})$} & \multirow[b]{2}{*}{$\mathrm{CV}(\%)$} \\
\hline & 150 & 300 & 450 & Média & \\
\hline \multicolumn{6}{|c|}{ Defeitos maiores (\%) } \\
\hline Soja & 2,56 & 2,50 & 2,62 & 2,56 & \\
\hline Salmão & 2,62 & 2,56 & 2,87 & 2,68 & \\
\hline Média & 2,59 & 2,53 & 2,75 & & 15,0 \\
\hline \multicolumn{6}{|c|}{ Defeitos menores $(\%)$} \\
\hline Soja & 1,87 & 1,81 & 1,06 & 1,58 & \\
\hline Salmão & 1,87 & 1,12 & 1,00 & 1,33 & \\
\hline Média & 1,87 & 1,46 & 1,03 & & 8,4 \\
\hline
\end{tabular}

Não houve diferença entre as médias pelo teste SNK $(\mathrm{P}>0,05)$.

Foi observado efeito da fonte de óleo $(\mathrm{P}<0,05)$ sobre o vigor espermático (Tab. 5), sendo maior no sêmen dos animais suplementados com óleo de salmão do que no dos tratados com óleo de soja.

Houve efeito $(\mathrm{P}<0,05)$ da adição de vitamina $\mathrm{E}$ à ração sobre a concentração espermática (Tab. 5), e não foi observada diferença quanto à fonte de óleo $(\mathrm{P}>0,05)$. As maiores concentrações ocorreram quando se utilizaram óleos com níveis de 150 e 450mg de vitamina E/kg de ração (Tab. 5). Em suínos, o efeito de micronutrientes sobre o desempenho reprodutivo não está esclarecido. Os resultados registrados neste estudo confirmam os obtidos por Marin-Guzman et al. (1997), que observaram melhora na qualidade espermática após a suplementação da dieta de suínos com selênio e vitamina E. Também Brzezinska-Slebodzinska et al. (1995) observaram aumento da concentração espermática no sêmen de suínos suplementados com vitamina E. Este foi um efeito, possivelmente, ligado às propriedades antioxidantes da vitamina $\mathrm{E}$.

Vale ressaltar que a vitamina E, lipossolúvel, é absorvida no intestino delgado juntamente com os lipídios da dieta e outras vitaminas lipossolúveis. A princípio, ela tem o papel de proteção do epitélio germinativo dos testículos contra degeneração, entretanto a suplementação dessa vitamina pode ocasionar redução das reservas de outras vitaminas lipossolúveis, por reduzir sua absorção e, consequentemente, prejudicar o desempenho reprodutivo animal (Zanini, 2001). A suplementação da vitamina E talvez possa ter dado proteção antioxidante aos espermatozoides de ambos os tratamentos. Estes resultados confirmam os já obtidos por Maldjian et al. (2005), que reportaram aumento da concentração espermática do sêmen de animais suplementados com DHA, sem qualquer adição extra de antioxidante, quando comparado com o sêmen dos animais que não receberam suplementação.

Com relação à concentração de antioxidantes totais (AOT) no sêmen, foi constatado efeito da fonte de óleo e dos níveis de vitamina $E$ $(\mathrm{P}<0,05)$. Após o desdobramento da interação, constatou-se que não houve diferença dos contrastes observados dentro da interação (P>0,05). Os animais suplementados com óleo de salmão apresentaram menor $(\mathrm{P}<0,05)$ concentração de AOT no sêmen do que os tratados com óleo de soja. Os resultados corroboram com os registrados por Zanini et al. (2003b), que, ao trabalharem com diferentes fontes de óleo na dieta de galos, constataram que o uso de ração com óleo de peixe resultou em menor AOT no sêmen. 
Tabela 5. Vigor espermático, concentração espermática e concentração de antioxidantes totais (AOT) nos espermatozoides no sêmen in natura de suínos, de acordo com a fonte de óleo e o nível de suplementação de vitamina $\mathrm{E}$

\begin{tabular}{|c|c|c|c|c|c|}
\hline \multirow{2}{*}{ Fonte de óleo } & \multicolumn{4}{|c|}{ Nível de vitamina $\mathrm{E}(\mathrm{mg} / \mathrm{kg})$} & \multirow[b]{2}{*}{$\mathrm{CV}(\%)$} \\
\hline & 150 & 300 & 450 & Média & \\
\hline & \multicolumn{4}{|c|}{ Vigor espermático } & \\
\hline Soja & 3,92 & 3,70 & 4,00 & $3,87^{\mathrm{b}}$ & \\
\hline Salmão & 4,00 & 4,00 & 4,07 & $4,02^{\mathrm{a}}$ & \\
\hline \multirow[t]{2}{*}{ Média } & 3,96 & 3,85 & 4,04 & & 8,75 \\
\hline & \multicolumn{4}{|c|}{ Concentração espermática (x10 células/mL) } & \\
\hline Soja & 424,6 & 401,5 & 439,2 & 421,7 & \\
\hline Salmão & 468,3 & 393,1 & 457,3 & 439,6 & \\
\hline \multirow[t]{2}{*}{ Média } & $446,5 \mathrm{~A}$ & 397,3B & $448,2 \mathrm{~A}$ & & 21,04 \\
\hline & \multicolumn{4}{|c|}{ Concentração de AOT (mmol/L) } & \\
\hline Soja & 21,66 & 21,79 & 22,24 & $21,89 a$ & \\
\hline Salmão & 21,18 & 21,14 & 21,12 & $21,14 b$ & \\
\hline Média $^{1}$ & $21,42 \mathrm{~B}$ & $21,46 \mathrm{~B}$ & $21,68 \mathrm{~A}$ & & 11,54 \\
\hline
\end{tabular}

Médias seguidas de letras distintas minúsculas na coluna ou maiúsculas na linha diferem entre si pelo teste SNK $(\mathrm{P}<0,05)$.

${ }^{1}$ Efeito linear $(\mathrm{P}<0,05)$.

Óleos de origem animal, como o de peixe, contêm elevado teor de PUFAs e baixo teor de vitamina $\mathrm{E}$ (tocoferol), e isto faz com que seja mais difícil a sua conservação mesmo em condições de refrigeração (Araújo, 1999). Bell et al. (1993) reportaram existir correlação negativa entre motilidade espermática total e peroxidação lipídica. Foi verificada correlação negativa entre motilidade e AOT nos espermatozoides nos tratamentos com óleo de soja $(r=-0,39)$ e óleo de salmão $(r=-0,45)$. Isto demonstra que os espermatozoides dos animais tratados com óleo de salmão, por serem ricos em PUFA e mais susceptíveis à peroxidação, apresentaram maior grau de peroxidação, consequentemente menor AOT (Tab. 5).

Maldjian et al. (2005) não encontraram melhora na qualidade dos parâmetros analisados dos espermatozoides de sêmen in natura após a suplementação com óleo de peixe na ração de suínos, sem qualquer adição extra de antioxidantes. Contudo, observaram-se resultados significativos do sêmen in natura e, com isso, pode-se aferir que parece existir um sinergismo entre antioxidantes e óleos de origem animal quando estes são adicionados à ração, e que, por isso, significantes melhoras da qualidade dos espermatozoides no sêmen in natura têm sido relatadas (Maldjian et al., 2003; Speake et al., 2003).

\section{CONCLUSÕES}

A adição de óleo de salmão na ração de suínos reprodutores não alterou os parâmetros físicos e morfológicos do sêmen in natura, com exceção do vigor, portanto esse óleo pode ser utilizado nas rações de suínos machos. Níveis de vitamina E de 150 e 450mg/kg de ração aumentaram a concentração espermática.

\section{AGRADECIMENTOS}

À FAPEMIG e ao CNPq, pelo apoio financeiro e à Perdigão S/A, pelo apoio logístico.

\section{REFERÊNCIAS BIBLIOGRÁFICAS}

AITKEN, R.J. A free radical theory of male infertility. Reprod. Fertil. Dev., v.6, p.19-24, 1994.

ARAÚJO, J.M.A. Química dos alimentos: teoria e prática. 2.ed. Viçosa: UFV, 1999. 416p.

BARTH, A.D.; OKO, R.J. Abnormal morphology of bovine spermatozoa. Ames: Iowa University, 1989. 285p.

BELL, M.; WANG, R.; HELLSTROM, W.J.G. et al. Effect of cryoprotective additives and cryopreservation protocol on sperm membrane lipid peroxidation and recovery of motile human sperm. J. Androl., v.14, p.472-478, 1993.

BRZEZINSKA-SLEBODZINSKA， E.; SLEBODZINSKI, A.B.; PIETRAS, B. et al. Antioxidant effect of vitamin E and glutathione on lipid peroxidation in boar semen plasma. Biol. Trace Elem. Res., v.47, p.69-74, 1995. 
CECIL, H.C.; BAKST, M.R. In vitro lipid peroxidation of turkey spermatozoa. Poult. Sci., v.72, p.1370-1378, 1993.

CONNOR, W.E.; LIN, D.S.; WOLF, D.P. et al. Uneven distribution of desmosterol and docosahexaenoic acid in the heads and tails of monkey sperm. J. Lipid Res., v.39, p.14041411, 1998.

CONQUER, J.A.; MARTIN, J.B.; TUMMON, I. et al. Effect of DHA supplementation on DHA status and sperm motility in asthenozoospermic males. Lipids, v.35, p.149-154, 2000.

FERREIRA NETO, J.M.; VIANA, E.S.; MAGALHÃES, L.M. Patologia clínica veterinária. Belo Horizonte: Rabelo Brasil, 1977. 279p

GULAYA, N.M.; TRONKO, M.D.; VOLKOV, G.L. et al. Lipid composition and fertile ability of human ejaculate, $U \mathrm{kr}$. J. Biochem., v.65, p.64-70, 1993.

HAMMERSTEDT, R.H. Maintenance of bioenergetic balance in sperm and prevention of lipid peroxidation: a review of the effect on design of storage preservation systems. Reprod. Fertil. Dev., v.5, p.675-690, 1993.

JONES, R.E.; PLYMATE, S.R. Synthesis of docosahexaenoyl coenzyme A in human spermatozoa. $J$. Androl., v.14, p.428-432, 1993.

JONES, R.E.; PLYMATE, S.R. Evidence for the regulation of fatty acid utilization in human sperm by docosahexaenoic acid. Biol. Reprod., v.39, p.76-80, 1988.

KING, G.J., MacCPHERSON, J.W. A comparison of two methods for boar semen collection. J. Anim. Sci., v.36, p.563$565,1973$.

KUMI-DIAKA, J. Subjecting canine semen to the hyposmotic test. Theriogenology, v.39, p.1279-1289, 1993.

MALDJIAN, A.; PIZZI, F.; GLIOZZI, T. et al. Changes in sperm quality and lipid composition during cryopreservation of boar semen. Theriogenology, v.63, p.411-421, 2005.

MALDJIAN, A.; PENNY, P.; NOBLE, R.C. Docosahexaenoic acid-rich marine oils and improved reproductive efficiency in pigs. In: De VRIESE, S.R.; CHRISTOPHE, A.B. (Eds). Male fertility and lipid metabolism. Champaign, Il: AOCS, 2003. p.60-72.

MANUAL para exame andrológico e avaliação de sêmen animal. 2.ed. Belo Horizonte: CBRA, 1998. 49p.

MARIN-GUZMAN, J.; MAHAN, D.C.; CHUNG, Y.K. et al. Effects of dietary selenium and vitamin $\mathrm{E}$ on boar performance and tissue responses, semen quality and subsequent fertilization rates in mature gilts. J. Anim. Sci., v.75, p.2994-3003, 1997.

MARIN-GUZMAN, J.; MAHAN, D.C.; WHITMOYER, R Effects of dietary selenium and vitamin $E$ on the ultrastructure and ATP concentration of boar spermatozoa, and the efficacy of added sodium selenite in extended semen on sperm motility. J. Anim. Sci., v.78, p.1544-1550, 2000.
MURGAS, L.D.S.; FIALHO, E.T.; OLIVEIRA, A.I.F. et al. Desempenho reprodutivo de varrões híbridos alimentados com óleo de soja como fontes de ácidos graxos. Cienc. Agrotec., v.25, p.1423-1434, 2001.

NISSEN, H.P.; KREYSEL, H.W. Polyunsaturated fatty acids in relation to sperm motility. Andrologia, v.15, p.264-269, 1983.

NIKI, E.; NOGUCHI, N.; GOTOH, N. Dynamics of lipid peroxidation and its inhibition by antioxidants. Biochem. Soc. Trans., v.21, p.313-317, 1993.

NUTRIENT requeriments of swine. 10.ed. Washington: National Academy of Science, 1998. 189p.

OLIW, E.H.; SPRECHER, H.W. Metabolism of polyunsaturated (n-3) fatty acids by monkey seminal vesicles: Isolation and biosynthesis of n-3 epoxides. Biochim. Biophys. Acta, v.1086, p.287-294, 1991.

PENNY, P.C.; NOBLE, R.C.; MALDJIAN, A. et al. Potencial role of lipids for the enhancement of boar fertility and fecundity. Pig News Inform., v.25, p.119-126, 2000.

SCOTT, J.W. Lipid metabolism of spermatozoa. J. Reprod. Fertil., v.18, suppl., p.65-76, 1973.

SIRRI, F.; MELUZZI, L.; PARDO RAMIREZ, C. et al. Fatty acid composition of lipid in eggs laid by hens fed diets supplemented with different fats. In: EUROPEAN SYMPOSIUM ON QUALITY OFF EGG AND EGG PRODUCTS, 6., 1995, Zaragoza. Proceedings...Zaragoza, 1995. p.411-500.

SISTEMA para análise estatística e genética - SAEG. Viçosa: UFV, 1997. 149p.

SPEAKE, B.K.; SURAI, P.F.; ROOKE, J.A. Regulation of avian and mammalian sperm production by dietary manipulation. In: De VRIESE, S.R.; CHRISTOPHE, A.B. (Eds). Male fertility and lipid metabolism. Champaign, Il: AOCS, 2003. p.96-117.

VAZQUEZ, J.M.; MARTINEZ, E.A.; GARCIA-ARTIGA, C. et al. Hypoosmotic swelling of boar spermatozoa compared to other methods for analysing the sperm membrane. Theriogenology, v.47, p.913-922, 1997.

ZANINI, S.F.; TORRES, C.A.A.; BRAGAGNOLO, N. et al. Fontes de óleos e níveis de vitamina E sobre desempenho produtivo e reprodutivo de galos. Rev. Bras. Cienc. Avic., supl.5, p.71, 2003a.

ZANINI, S.F.; TORRES, C.A.A.; NEURA, B. et al. Evaluation of the ratio of w6:w3 fatty acids and vitamin $\mathrm{E}$ levels in the diet on the reproductive performance of cockerels. Arch. Tierernahr., v.57, p.429-442, 2003 b.

ZANINI, S.F. Fontes de óleo e níveis de suplementação de vitamina $E$ na ração sobre o desempenho produtivo $e$ reprodutivo de galos leves. 2001. 139f. Tese (Doutorado) Universidade Federal de Viçosa, Viçosa, MG. 